\title{
PARACOCCIDIOIDOMICOSIS SUBAGUDA GANGLIONAR PRESENTACIÓN DE UN CASO CLINICO EN PEDIATRIA
} JORGE BELTRÁN ${ }^{1}$, XIMENA ARENAS ${ }^{1}$, LAURA TORRES ${ }^{2}$, ISABEL HURTADO ${ }^{3,4}$, EdUARDO LOPEZMEDINA $^{3,4}$, ALEXANDRA SIERRA ${ }^{3,4}$, MARIA TERESA RAMIREZ ${ }^{5}$, LUISA VALENCIA ${ }^{5}$, PIO LOPEZ ${ }^{3,4}$

\section{RESUMEN}

La paracoccidioidomicosis, enfermedad sistémica fungémica, es causada por Paracoccidioides brasiliensis. Se produce por la inhalación de conidias, conduciendo a una infección pulmonar transitoria que por lo general, se autoresuelve. En jovenes puede progresar a una forma aguda o subaguda. Generalmente quienes la padecen son agricultores, ya que los principales lugares donde se localiza el gérmen es en los cultivos de café, cacao y algodón. Las manifestaciones clinicas pueden ser en piel, mucosas, sistema reticuloendotelial, pulmones, y glandula suprarrenal, entre otros. Su manejo es con antimicóticos por tiempo prolongado y por lo general se requieren combinaciones de los mismos. Se presenta el caso de un adolescente con paracoccidioidomicosis subaguda.

\section{Palabras claves: paracoccidioidomicosis, adolescentes}

\section{INTRODUCCIÓN}

La paracoccidioidomicosis es una micosis sistémica causada por el hongo Paracoccidioides brasiliensis. Una gran proporción de los casos ocurren en brasil pero se han reportando en toda America Latina ${ }^{1}$. La infeccion se produce principalmente por inhalación de conidias, lo que conduce a una infección pulmonar transitoria que frecuentemente autoresuelve. En los pacientes jovenes puede progresar a una forma aguda o subaguda, mientras que en pacientes adultos se puede desarrollar una forma crónica con largos periodos de latencia. Los principales nichos del germen son los cultivos de café, cacao y algodón, por lo que las infecciones usualmente se deben a exposiciones ocupacionales en agricultores ${ }^{2}$. Las manifestaciones clinicas son muy variadas y dependen del organo comprometido, puediendo producir afección de piel y mucosas, sistema reticuloendotelial, pulmones, glandula suprarrenal, entre otros ${ }^{3,4}$. El tratamiento con antimicoticos es por largo plazo, y generalmente es

${ }^{1}$ Departamento de Pediatría. Universidad del Valle, Cali, Colombia

${ }^{2}$ Facultad de Medicina. Universidad del Valle, Cali, Colombia

${ }^{3}$ Departamento de Pediatría. Sección de Infectología Pediátrica,

Universidad del Valle, Cali, Colombia

${ }^{4}$ Centro de Estudios en Infectología Pediátrica. CEIP. Cali, Colombia

${ }^{5}$ Departamento de Patología, Universidad del Valle. Cali, Colombia

Recibido para publicación: enero 15, 2015

Aceptado para publicación: agosto 14, 2015

\section{SUMMARY}

Paracoccidioidomycosis, fungic systemic disease, is caused by Paracoccidioides brasiliensis. It is caused by the inhalation of conidia, leading to a transient lung infection usually is autoresuelve. In adolescents can progress to acute or subacute. Usually sufferers are farmers, as the main places where the germ is located is in crops of coffee, cocoa and cotton. Clinical manifestations may be in skin, mucosa, reticuloendothelial system, lungs, and adrenal glands, among others. Its handling is with antifungal prolonged and usually combinations thereof are required. The case of adolescent with subacute paracoccidioidomycosis is presented.

Key words: Paracoccidioidomycosis, Adolescents

necesario una combinación de estos ${ }^{3}$. A continuacion se expondrá el caso de un paciente adolescente con paracoccidioidomicosis subaguda y se analizará a la luz de la literatura.

\section{CASO CLINICO}

Paciente masculino de 10 años de edad, procedente del área rural del municipio de Yotoco, departamento del Valle del Cauca, Colombia. Consulta por cuadro clínico de 15 dias de evolución consistente en astenia, adinamia, pérdida de peso no cuantificado y fiebre alta persistente, asociado a presencia de masa cervical anterior izquierda, de crecimiento progresivo, no dolorosa.

Dentro de sus antecedentes epidemiologicos, se destaca vivienda con piso de tierra y cemento, paredes en tablas de madera. La madre y uno de sus hermanos son recolectores de café, encontrando que el paciente los asiste ocacionalmente en esta labor.

En el examen físico de ingreso se evidencia paciente con signos vitales: Frecuencia cardiaca: 135 latidos por minuto Frecuencia respiratoria: 24 respiraciones por minuto PA: $100 / 60 \mathrm{mmHg} \mathrm{T}{ }^{\circ}: 36,4^{\circ} \mathrm{C}$ Peso: $30 \mathrm{~kg}(-1$ desviación estándar) Talla: $136 \mathrm{~cm}$ (-1 desviación estándar) IMC: 16.3. Se encuentra masa cervical 
anterior izquierda ubicada en zona 2 de aproximadamente $5 \times 7 \mathrm{~cm}$ de longitud, que se extiende hacia region posterior, de bordes mal definidos sin cambios de inflamación local, no móvil, indolora, adherida a planos profundos Figura 1. Se palpan múltiples adenopatías cervicales anteriores y posteriores, axilares e inguinales, la mayor de $1 \mathrm{~cm}$ de longitud, todas indoloras y sin cambios de inflamación local. Se evidencia hepatomegalia de 7 centimetros por debajo del reborde costal derecho con linea medio clavicular, no dolorosa, con higado de consistencia normal, sin esplenomegalia. Resto del examen fisico sin alteraciones.

Examenes de laboratorio de ingreso se muestran en tabla 1. Se hace diagnostico de adenitis bacteriana y se inicia manejo con clindamicina intravenosa (IV) $40 \mathrm{mg} / \mathrm{kg} / \mathrm{día}$ dividido cada 6 horas. Durante la hospitalización el paciente persiste con fiebre, aumento del tamaño de las adenopatias y leucocitosis con neutrofilia persistente y aumento en la proteina $\mathrm{C}$ reactiva por lo que se avanza en la exploración clinica.

Se toma radiografia de torax que muestra masa paracardiaca derecha. Por hallazgos de radiografía de tórax se ordena tomografía axial computarizada cervical, torácica y abdominal que evidencia múltiples adenopatías cervicales, torácicas y abdominales Figura 2. Se ordena biopsia de ganglio cervical anterior izquierdo en la que se encuentra inflamación granulomatosa crónica y presencia de estructuras micóticas, con diagnostico por patologia de paracoccidioidoicosis Figura 3. Se inicia manejo con Itraconazol $10 \mathrm{mg} / \mathrm{kg} /$ día y trimetoprim-sulfametoxazol $16 \mathrm{mg} / \mathrm{kg} /$ día con lo cual desaparece la fiebre y las adenopatías disminuyen progresivamente de tamaño. Se realizo visita de control a las 12 semanas posterior al inicio del tratamiento encontrandose paciente en buenas condiciones generales, con desaparicion total de las adenopatias, adecuada ganancia de peso y sin aparicion de nuevas lesiones.

\section{DISCUSIÓN}

La paracoccidioidomicosis es una micosis poco comun producida por Paracoccidioides brasiliensis. Es un hongo de la familia Ajellomycetaceae, orden Onygenales, y presenta varias especies filogenéticas: PS1, PS2, PS3, PS4. Se considera un hongo dimorfico, ya que a temperatura menor de $28^{\circ} \mathrm{C}$ se comporta como moho (hifas como forma infectante), y a temperaturas de $35-37^{\circ} \mathrm{C}$ se comporta como Levadura (forma patógena multigemante) $)^{2}$.

Se encuentra distribuido alrededor de toda Suramérica, desde México a Argentina. Se ha descrito en áreas tropicales y subtropicales con altitud 1000-1499 metros sobre el nivel del mar, con altas precipitaciones de lluvia y presencia de bosques húmedos, y se ha relacionado además con cultivos agrícolas de café, tabaco, Algodón, y caña de azúcar ${ }^{4-5}$. Los estudios en Colombia demuestran que las zonas de Santander aledañas al río Suárez, el Suroeste de Antioquia, las estribaciones de la Sierra Nevada de Santa Marta y el Valle del Cauca son los sitios de donde provienen la mayoría de los $\operatorname{casos}^{4,6,7}$. Nuestro paciente cumplía con algunas de estas características epidemiológicas (zona de procedencia y cercanía con cultivos de café) lo que pudo orientar hacia el diagnostico.

En Colombia se calcula una prevalencia (mediante pruebas serológicas) de 0,5-2.2/millón de personas. Se estima que el $9 \%$ de la población ha estado en contacto con este hongo ${ }^{4,5}$. Se ha encontrado co-infección con VIH-SIDA en un $1,4 \%$ y TBC en $8 \%$. Es raro en niños y adolecentes (menos del $9 \%$ de los casos) con relación Hombre: Mujer de 30: 1, a diferencia de los niños: niñas 1-2:1. Estas diferencias demográficas se explican por la mayor actividad del hombre en el campo, asociado a un efecto protector del beta estradiol, el cual interviene en la transformación del hongo a su forma propagativa $^{2,6}$.

La forma principal de infección es por inhalacion de conidias, sin embargo, pocos sujetos infectados desarrollan enfermedad, ${ }^{8}$, lo cual depende de la inmunidad del huesped, la edad y el inoculo micotico ${ }^{2}$. La enfermedad se dividide en 4 categorias según las manifestaciones clinicas:

1.Infección subclínica: Proceso latente, clínicamente asintomatico que puede permanecer así años después del contacto inicial con el hongo. Posteriormente puede evolucionar a enfermedad progresiva, clínicamente manifiesta ${ }^{2}$.

2.Forma Residual: Presencia de tejido fibroso en los organos previamente infectados, principalmente en los pulmones, pero también en las glándulas suprarrenales y mucosas ${ }^{2}$.

3.Enfermedad Aguda / Subaguda: Proceso con 
manifestaciones graves, con evidencia de participación de múltiples órganos, como ganglios linfáticos, hígado, bazo, lesiones de la piel, pulmones. El compromiso de este ultimo no suele revelar anormalidades radiográficas, pero si manifestaciones inespecificas como tos cronica, espectoracion, hemoptisis, disnea. En ocaciones se documenta derrame mediastinal, detectando $P$. brasiliensis en muestras respiratorias, asi como sintomas constitucionales: dolor abdominal, fiebre, pérdida de peso, hipercalcemia ${ }^{2,9}$. Esta forma generalmente se diagnostica en niños, adultos jóvenes y pacientes con alteraciones inmunológicas como VIH/SIDA.

4.Enfermedad Crónica Progresiva: La más común (90\%) de las presentaciones clínicas. Es diagnosticada principalmente en pacientes de edad avanzada y se caracteriza por el compromiso importante de pulmón y lesiones frecuentes en mucosas, piel y glandulas suprarrenales. Se manifiestan clínicamente por síntomas constitucionales (fiebre, astenia, malestar general, pérdida de peso), asociado a la clinica según el organo afectado ${ }^{2}$.

Las alteraciones paraclinicas iniciales incluyen anemia, leucocitosis con neutrofilia y eosinofilia y algunas veces trombocitopenia. La eosinofilia aparece en más de la mitad de los pacientes y puede ser intensa en algunos casos. Otras manifestaciones de laboratorio son hipoalbuminemia grave, hipergammaglobulinaemia, elevación de las enzimas hepáticas y bilirrubinas (predominio fracción conjugada), así como elevacion de reactantes de fase aguda. Todos estos estudios son inespecificos y no sugieren etiologia específica , razón por la cual se deben realizar otros estudios como cultivos para hongos y pruebas de histopatologia tendientes a identificar el diagnostico. Es importante tener en cuenta que la serologia no es adecuada para hacer diagnostico de enfermedad ya que solo nos indica exposicion al hongo, aunque puede ser util para monitorizar la respuesta inmune del paciente durante el tratamiento.

En nuestro caso, se recurrió a una biopsia ganglionar donde la histopatología y el cultivo hicieron el diagnostico final de esta infección al revelar en el tejido celulas de forma levaduriforme con apariencia de "rueda de timón de barco". Es importante tener en cuenta que la visualización del hongo puede realizarse en diversas muestras clínicas frescas (esputo, raspaduras de lesiones mucocutáneas, aspirado ganglionar) y / o en material de biopsia (piel, mucosas, ganglios linfáticos etc.) utilizando una tinción con Gomori-Grocott o ácido periódico de Schiff(PAS) ${ }^{10}$.

En cuanto al aislamento microbiologico, este se debe realizar en cultivos con medios apropiados (Sabourand, Mycosel, Difco) para revelar el crecimiento de hongos (hifas) a temperatura ambiente después de 20 a 30 días. A $37^{\circ} \mathrm{C}$, el crecimiento de levaduras se tarda entre $10 \mathrm{y}$ 12 dias de incubacion, lo que confirma el dimorfismo térmico de esta microorganismo ${ }^{10,11}$.

Las pruebas serológicas específicas (inmunodifusión doble, contrainmunoelectroforesis, inmunofluorescencia indirecta, inmunoensayo enzimático, y de inmunotransferencia, detectando glicoproteina 43, sensibilidad y especificidad 85$100 \%$ ) son importantes como una ayuda diagnóstica, y evaluación de la respuesta del huésped al tratamiento. Los resultados falsos negativos pueden ocurrir en formas localizadas de la micosis o en huéspedes inmunodeprimidos, mientras que resultados falsos positivos pueden ocurrir en histoplasmosis y $\operatorname{aspergilosis}^{9,12}$.

La terapia antifúngica es la base para la mejora del paciente. Existen tres grupos de medicamentos disponibles: sulfonamidas, anfotericina $\mathrm{B}$, y derivados azólicos, los cuales, dependiendo de la clinica y las caracteristicas del paciente (tipo de infección, edad, comorbilidades como VIH, tuberculosis y cancer) se combinarán y se administrarán en esquema endovenoso inicial, para posterior continuacion oral, cumpliendo esquemas de largo tiempo de 6 meses hasta 1 año. Se han demostrado mayores tasas de recidivas con el uso de anfotericina $\mathrm{B}$, mientras que con la terapia combinada las tasas de éxito son las mas prometedoras $^{2,13,14}$. A nuestro paciente se le instauro tratamiento con itraconazol combinado con trimetoprim-sulfametoxasol por tratarse de un paciente joven, con enfermedad diseminada para así tener mayor probabilidad de éxito.

Uno de los escenarios a considerar es la presencia de alguna alteración inmune, ya que los mecanismos de defensa principalmente la inmunidad innata (polimorfonucleares neutrofilos, macrófagos alveolares, células dendríticas, celulas natural killer) que genera una respuesta Th1 detienen la progresión de 
la infección, por tanto, no todas las personas desarrollan la enfermedad ${ }^{10}$. En este orden de ideas, seria importante realizar estudios para inmunodeficiencias a estos pacientes en busca de factores predisponentes para la enfermedad, todos estos exámenes fueron normales en nuestro paciente.

En conclusión, la paracoccidioidomicosis en nuestro medio es una enfermedad de incidencia no calculada exactamente por su bajo índice de sospecha y poca notificación de la misma. Es una micosis sistémica que afecta principalmente los pulmones y puede comprometer mucosas, piel, sistema reticuloendotelial, hueso y glándulas suprarrenales. La presentación mas común en pacientes adolescentes es la forma aguda/subaguda, encontrándose compromiso principalmente del sistema reticuloendotelialganglionar, por lo cual, dentro del diagnostico diferencial de las adenomegalias debe tenerse en cuenta esta infección. Es importante incluir esta patologia como diagnostico diferencial de las adenopatias, principalmente en pacientes que residan en zonas de alto riesgo, sin descartar otras patologias prevalentes como infecciones bacterianas, tuberculosis infecciones virales y patologias propias del sistema hematopoyetico, especialmente en aquellos casos que no mejoren de forma apropiada con antibacterianos.

No diagnosticamos lo que no sospechamos, y no sospechamos lo que no conocemos.

\section{REFERENCIAS}

1. Bocca A, Amaral A, Teixeira M, Sato P, Shikanai-Yasuda M, Soares-Felipe M. Paracoccidioidomycosis: ecoepidemiology, taxonomy and clinical and therapeutic issues. Future Microbiol 2013; 8,9: 117-119

2. Restrepo A., Tobón A, Cano L. Paracoccidioidomycosis. In: Mandell. Principles and Practice of Infectious Diseases, 8th edition edition

3. Restrepo A, McEwen J, Castañeda E. The hábitat of Paracoccidiodes brasiliensis: how far from solving the riddle? Med Mycol 2001; 39: 232-241

4. Calle D, Rosero D, Orozco L, Restrepo A. Paracoccidioidomycosis in Colombia: an ecological study. Epidemiol Infect 2001; 126 309-315

5. Torrado E, Castañeda E, De la Hoz F, Restrepo A. Paracoccidioidomicosis: definición de las áreas endémicas de Colombia. Biomedica 2000;20: 327-334

6. Bellissimo-Rodrigues F, Machado A, Martinez R. Paracoccidioidomycosis: epidemiological features of a 1,000 - cases series from a hyperendemic area on the southeast of Brazil. Am J Trop Med Hyg 2011; 85: 546-550

7. Restrepo A. Coccidioides immitis y Paracoccidioides brasiliensis: dos hongos patógenos restringidos al Continente Americano. Rev Acad Colomb Cienc 2006; 30: 367-386

8. Shikanai-Yasuda M, Telles Filho Fde Q, Mendes RP, Colombo A, Moretti ML. Consenso em Paracoccidioidomicose. Rev Soc Bras Med Trop 2006; 39 : 297-310

9. Cano L, González A, Lopera D. Pulmonary paracoccidioidomycosis: clinical, immunological and histopathological aspects. In: Malcolm-Irusen E. Lung Diseases: Selected State of the Art Reviews. Editorial Rijeka, Croatia: InTech 2012

10. Marques S. Paracoccidioidomycosis: epidemiological, clinical, diagnostic and treatment updating. An Bras Dermatol 2013; 88: 700-711

11. Ferreira M. Paracoccidioidomycosis. Paediatric Respiratory Reviews 2009; 10: 161-165

12. Fortes M, Miot H, Kurokawa C, Marques M, Marques S. Immunology of paracoccidiodomycosis. An Bras Dermatol 2011; 86: 516-525

13. Nogueira M, Andrade G, Tonelli E, Diniz S, Goes A, Cisalpino P. Laboratory evolutive aspects of children under paracoccidioidomycosis treatment. Rev Soc Bras Med Trop 2006; 39: 478-483

14. Froes $\mathrm{H}$, Coligiorne RB. Fundamentals of paracoccidioidomycosis treatment. Drug Develop Res 2011; 72: 528-537 